\title{
Hopping process of bound excitons under an energy gradient
}

\author{
Gwénolé Jacopin, ${ }^{\text {a) }}$ Mehran Shahmohammadi, Jean-Daniel Ganière, and Benoît Deveaud \\ Laboratory of Quantum Optoelectronics, Ecole Polytechnique Fédérale de Lausanne (EPFL), 1015 Lausanne, \\ Switzerland
}

(Received 16 December 2013; accepted 11 January 2014; published online 29 January 2014)

\begin{abstract}
We report on the mechanism of hopping for bound excitons under an energy gradient. By means of a Monte-Carlo simulation, we show that this mechanism explains the movement of bound excitons observed experimentally. We show that the speed of the excitons decreases quickly with temperature. Thanks to an effective medium approximation, we deduce an analytical model to estimate the average speed at $\mathrm{T}=0 \mathrm{~K}$. Finally, we compare our simulations results to the speed observed in bent $\mathrm{ZnO}$ wires and find a good agreement between theory and experiments. (C) 2014 AIP Publishing LLC. [http://dx.doi.org/10.1063/1.4863319]
\end{abstract}

The ability to tune the strain in different structures is interesting for many applications such as the enhancement of the mobility in transistors ${ }^{1}$ or the engineering of the optical polarization properties of heterostructures. ${ }^{2,3}$ Recently, it has been shown that it is possible to design nano/microstructures to obtain a strain gradient and control the motion of excitons. ${ }^{4,5}$ In particular, bent nanowires have gained considerable attention because of the possibility to tune the bandgap without plastic relaxation..$^{6-8}$ For instance, it has been recently demonstrated that excitons can move because of the strain gradient induced by the bending of a wire. ${ }^{9}$ However, at $\mathrm{T}=10 \mathrm{~K}$ at which the experiments are performed, the photoluminescence of $\mathrm{ZnO}$ wires is dominated by the emission coming from the donor bound exciton $\left(\mathrm{D}^{\circ} \mathrm{X}\right)$. Thus, experimental evidence indicates that donor bound excitons are drifted by the strain gradient, against simple views implied by their name: "donor bound exciton."

The motion of bound excitons has already been observed in inhomogeneous materials such as GaAsP:N, ${ }^{10,11}$ InGaN, ${ }^{12}$ and $\mathrm{MgZnO}$ alloys. ${ }^{13}$ In these random alloys, the transfer of such excitons from one donor to another is driven by the tunneling of exciton to lower states. The same mechanism has been observed and successfully described by a stochastic model of exciton transfer in homogeneous $\mathrm{GaP}$ with well-defined impurities levels. ${ }^{14}$ However, in such samples, the random distribution of lowest states makes it difficult to obtain an accurate estimation of the characteristic time for the exciton to jump from one donor to the other.

The strain-induced energy gradient in bent micro/nanowires directly builds a "donor ladder" as observed in the scheme of Figure 1. Indeed, because of the continuous change of bandgap energy, the relative positions of donors are sorted by energy along the strain gradient. This arrangement of donors gives the possibility to get an accurate estimation of the hopping rate of excitons, unlike in the case of inhomogeneous materials.

In this Letter, we propose a model for the hopping process of donor bound excitons in the presence of an energy gradient. We compute the exciton dynamics by means of Monte-Carlo (MC) simulations taking into account the

a) gwenole.jacopin@epfl.ch probability for the transfer of an exciton from a donor to another. We compare the case of zero temperature to the case of a nonzero temperature and show that the mean speed should decrease with temperature against common wisdom. Then, by assuming an effective medium, we deduce an analytical solution to describe the hopping process at $\mathrm{T}=0 \mathrm{~K}$ and we discuss the effect of the temperature under this assumption.

The hopping rate $\nu_{i j}$ from one donor $i$ at an energy $E_{i}$ to another one $j$ at an energy $E_{j}$ is given by ${ }^{15,16}$

$$
\left\{\begin{array}{lll}
\nu_{i j}=\nu_{0} e^{-2 \frac{R_{i j}}{a_{0}}} & \text { if } & E_{j}<E_{i} \\
\nu_{i j}=\nu_{0} e^{-2 \frac{R_{i j}}{a_{0}}} e^{\frac{E_{i}-E_{j}}{k_{B} T}} & \text { if } & E_{j}>E_{i},
\end{array}\right.
$$

where $\nu_{0}$ is a constant corresponding to the typical time constant of the process, $a_{0}$ is the Bohr radius of the exciton bound to a donor, $k_{B}$ is the Boltzmann constant, $R_{i j}$ is the distance between the two donors, and $T$ is the lattice temperature. Equation (1) illustrates that the hopping rate decreases exponentially with the distance between donors and that the rate for hopping to a higher energy state is thermally activated. As a matter of fact, if $T=0 \mathrm{~K}$, only transitions to lower states are allowed. In the context of an energy

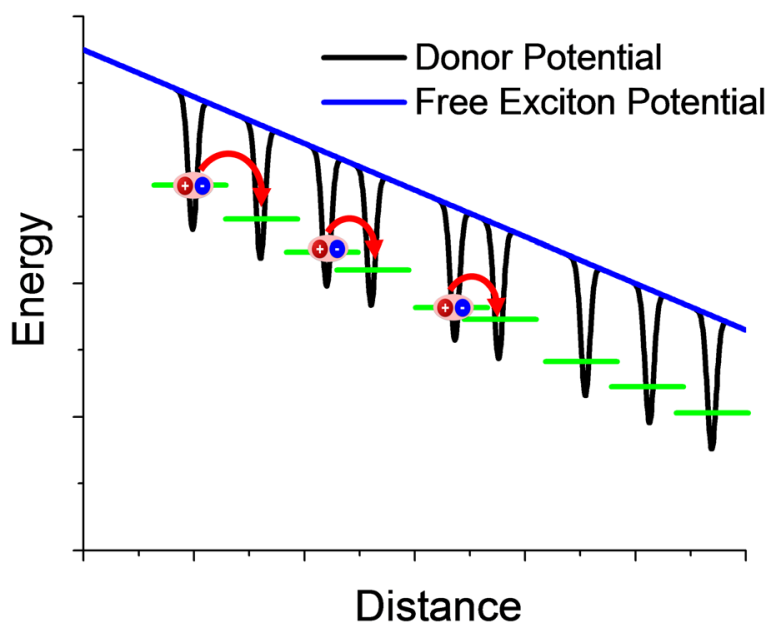

FIG. 1. Scheme of hopping mechanism of bound exciton in an energy gradient. 
gradient, it means that excitons can move only towards the low energy side.

In order to determine the mean speed of bound excitons in an energy gradient, we carry out MC simulations using the following model. First, we define within a finite 3D volume, the random positions of $N$ donors. We then arbitrary set the energy gradient along the z-axis. To keep the model as simple as possible, we consider a homogeneous energy gradient $E_{\sigma}$ defined as $E_{\sigma}=\frac{\Delta \mathrm{E}}{\Delta z}$ where $\Delta \mathrm{E}$ is the energy difference over the distance $\Delta \mathrm{z}$. This assumption is in good agreement with the experimentally observed strain gradient in bent $\mathrm{ZnO}$ wires. ${ }^{8,9}$ Then, we select one donor and we calculate all the hopping rates $\nu_{i j}$ from this donor to any other. The sum over all the donors gives us the transition time $\tau_{i}$ for the hopping to the next donor

$$
\frac{1}{\tau_{i}}=\sum_{j \neq i} v_{i j} .
$$

In order to choose the next donor, we choose randomly $\Gamma$, a number between 0 and $1 / \tau_{i}$ and use the cumulative distribution function $f_{\text {cum }}$, defined as

$$
f_{\text {cum }}(j)=\sum_{\substack{k=1 \\ k \neq i}}^{j} v_{i k} .
$$

If $f_{\text {cum }}(j)<\Gamma<f_{\text {cum }}(j+1)$, then the next donor where the exciton will be bound is the donor $j$. This method allows to respect the relative probability of each transition: The most probable donor has the maximum probability to be chosen. By this means, we virtually reproduce the "choice" of the exciton. We repeat this procedure until the exciton has travelled along the $\mathrm{z}$-axis over a given distance $d \gg \frac{1}{\sqrt[3]{N_{d}}}$, where $N_{d}$ is the donor concentration. By knowing the distance $d$ and the time $\tau_{\text {tot }}$ to travel over this distance, we are able to estimate the mean speed of the exciton along the energy gradient. Figure 2 shows an example of trajectory of an exciton in an energy gradient at $T=0 \mathrm{~K}$ for a donor concentration of $N_{d}=10^{18} \mathrm{~cm}^{-3}$ and a Bohr radius $a_{0}=5 \mathrm{~nm}$. Thanks to the energy gradient and the zero temperature, the exciton always "chooses" a donor with a larger z-position. For this specific case, assuming $\nu_{0}=10^{13} \mathrm{~s}^{-1},{ }^{11}$ we deduce an average speed of the exciton along the $\mathrm{z}$-axis of $5.4 \mathrm{~nm} / \mathrm{ps}$.

Since we are dealing with a random process, we have to repeat the process for a large number of excitons to obtain an average speed of donor bound excitons in an energy gradient. Figure 3(a) shows the average speed of excitons, resulting from MC simulations for a domain of $L_{x}=300 \mathrm{~nm}, L_{y}=300$ $\mathrm{nm}, L_{z}=300 \mathrm{~nm}$, and 10000 trials for $T=0 \mathrm{~K}$. We show the results as a function of the Bohr radius of the donor bound exciton. Here, it is worth noticing that for $T=0 \mathrm{~K}$, the average speed does not depend on the value of $E_{\sigma}$. Indeed, in this case, the hopping is only allowed towards lower energy states and does not depend on the energy difference (Eq. (1)). This is the reason why we prefer to define an average speed rather than an effective mobility of bound excitons.

In Figure 3(a), we observe a strong dependence of the average speed on the Bohr radius. This could be easily understood since the hopping rate exponentially depends on Bohr radius (Eq. (1)). For $N_{d}=10^{18} \mathrm{~cm}^{-3}$ and $\nu_{0}=10^{13}$ $\mathrm{s}^{-1}$, the average speed changes from $v=3.2 \times 10^{-3} \mathrm{~nm} / \mathrm{ps}$ for $a_{0}=2 \mathrm{~nm}$ to $v=590 \mathrm{~nm} / \mathrm{ps}$ for $a_{0}=15 \mathrm{~nm}$. We plot in Figure 3(b) the dependence of the exciton speed with respect to the temperature. For finite temperatures, the value of $E_{\sigma}$ plays then an important role because the probability that an exciton goes to a higher energy state is proportional to $e^{\frac{E_{\sigma}\left(z_{i}-z_{j}\right)}{k_{B} T}}$. The value $R_{h i g h}=\frac{E_{\sigma}}{k_{B} T \times \sqrt[3]{N_{d}}}$ gives information about the ability of an exciton to go to higher energy states. If $R_{\text {high }} \gg 1$, then excitons travel only to lower states. In contrast, if $R_{\text {high }} \ll 1$, excitons do not "feel" the energy gradient and the probability to choose the higher or lower energy side is equal. This behavior explains why, in Figure 3(b), the speed decreases with temperature. Indeed, excitons travel not only to $\mathrm{z}>0$ but also towards $\mathrm{z}<0$, thus, it takes more
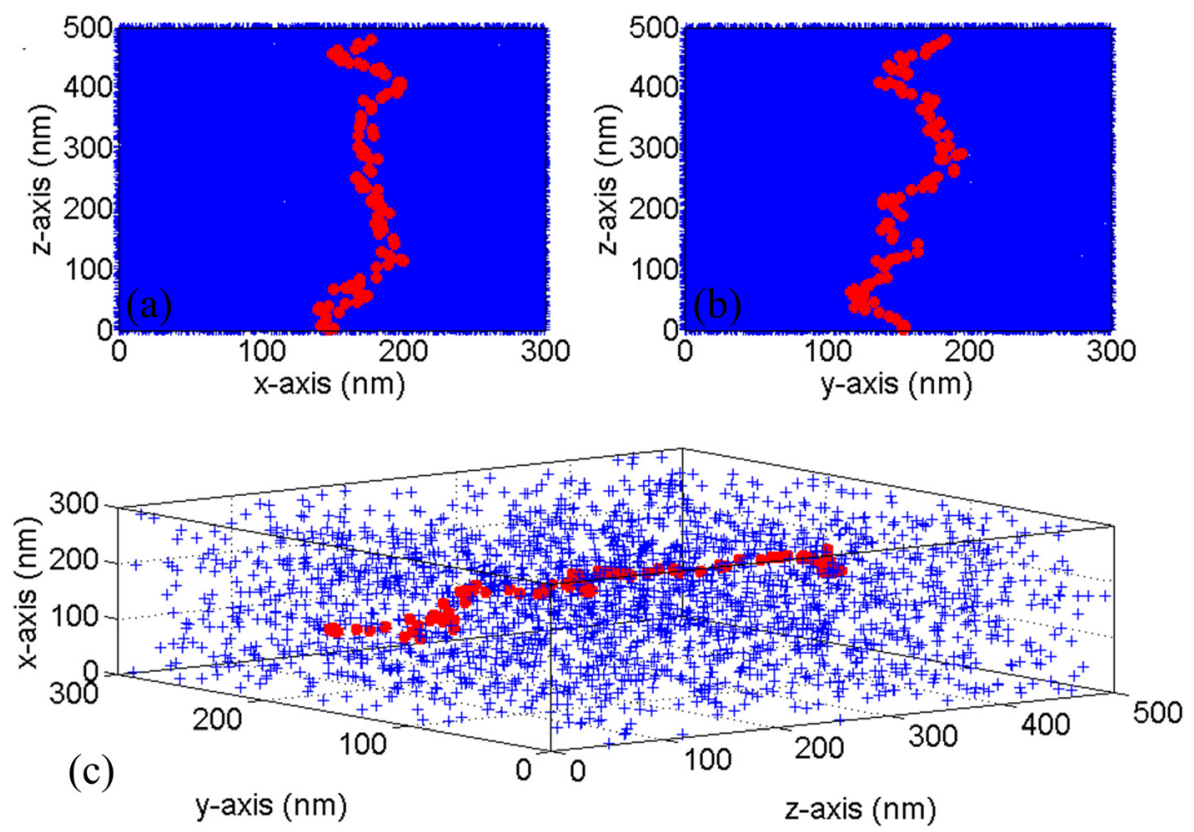

FIG. 2. Example of the trajectory of an exciton in an energy gradient along the $\mathrm{z}$-axis at $\mathrm{T}=0 \mathrm{~K}$ for a donor concentration of $\mathbf{N}_{\mathbf{d}}=10^{18} \mathrm{~cm}^{-3}$ and a Bohr radius $\mathbf{a}_{0}=5 \mathrm{~nm}$. Blue crosses show the donor positions and red dots show the donors where the exciton actually went. (a) $x-z$ projection. (b) y-z projection. (c) 3D view. 

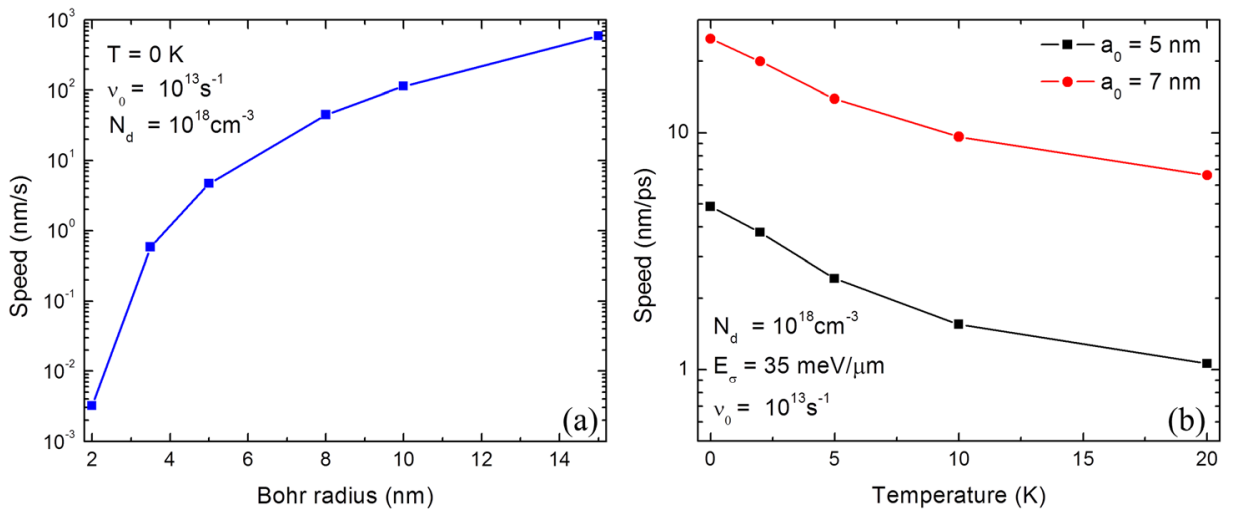

FIG. 3. (a) Average speed of exciton at $T=0 \mathrm{~K}$ as a function of the Bohr radius. (b) Average speed of exciton as a function of temperature for two different Bohr radius: $\mathrm{a}_{0}=5 \mathrm{~nm}$ (black squares) and $\mathrm{a}_{0}=7 \mathrm{~nm}$ (red dots).

TABLE I. Average speed of exciton estimated by $\mathrm{MC}$ at $\mathrm{T}=0 \mathrm{~K}$ and $\mathrm{T}=20 \mathrm{~K}$ for two Bohr radii of $a_{0}=5 \mathrm{~nm}$ and $a_{0}=7 \mathrm{~nm}$.

\begin{tabular}{lcr}
\hline \hline & $a_{0}=5 \mathrm{~nm}$ & $a_{0}=7 \mathrm{~nm}$ \\
\hline $\mathrm{T}=0 \mathrm{~K}$ & $4.9 \mathrm{~nm} / \mathrm{ps}$ & $24.9 \mathrm{~nm} / \mathrm{ps}$ \\
$\mathrm{T}=20 \mathrm{~K}$ & $1.1 \mathrm{~nm} / \mathrm{ps}$ & $6.6 \mathrm{~nm} / \mathrm{ps}$ \\
\hline \hline
\end{tabular}

time to travel (in mean value) along the $\mathrm{z}$-axis $(\mathrm{z}>0)$. For an energy gradient of $35 \mathrm{meV} / \mu \mathrm{m},{ }^{9}$ as shown in Table I, the simulations give a value of $v=1.6 \mathrm{~nm} / \mathrm{ps}$ (resp. $v=9.6$ $\mathrm{nm} / \mathrm{ps}$ ) for $T=10 \mathrm{~K}$ and $a_{0}=5 \mathrm{~nm}$ (resp. $a_{0}=7 \mathrm{~nm}$ ). Experimentally, we deduce a speed around $5 \mathrm{~nm} / \mathrm{ps}$ at $10 \mathrm{~K}$.
In the following, we will consider the approximation of an effective medium. Under this assumption (valid if $a_{0} \sqrt[3]{N_{d}} \gg 1$ ), instead of considering a discrete nature of donors, we consider a homogeneous density of donors. Hence, we can replace the discrete summation by a triple integral. The mean transition time between donors at a position $x=x_{0}, y=y_{0}$, and $z=z_{0}$ becomes then

$$
\frac{1}{\tau}=\sum_{j=1}^{\infty} v_{i j} \approx \int_{-\infty}^{\infty} \int_{-\infty}^{\infty} \int_{-\infty}^{\infty} N_{d} v(x, y, z) \mathrm{d} x \mathrm{~d} y \mathrm{~d} z
$$

with

$$
\begin{cases}v(x, y, z)=\nu_{0} \mathrm{e}^{-2 \frac{\sqrt{\left(x-x_{0}\right)^{2}+\left(y-y_{0}\right)^{2}+\left(z-z_{0}\right)^{2}}}{a_{0}}} & \text { if } \quad z>z_{0} \\ v(x, y, z)=\nu_{0} \mathrm{e}^{-2 \frac{\sqrt{\left(x-x_{0}\right)^{2}+\left(y-y_{0}\right)^{2}+\left(z-z_{0}\right)^{2}}}{a_{0}}} e^{-\frac{E_{\sigma}\left(z_{0}-z\right)}{k_{B} T}} & \text { if } \quad z<z_{0} .\end{cases}
$$

In addition, the mean hopping distance $d$ between two donors along the z-axis becomes

$$
d=\tau \int_{-\infty}^{\infty} \int_{-\infty}^{\infty} \int_{-\infty}^{\infty}\left(z-z_{0}\right) N_{d} v(x, y, z) \mathrm{d} x \mathrm{~d} y \mathrm{~d} z .
$$

Finally, we deduce the speed $v$ of bound excitons in such a medium

$$
v=\frac{\mathrm{d}}{\tau}=\int_{-\infty}^{\infty} \int_{-\infty}^{\infty} \int_{-\infty}^{\infty}\left(z-z_{0}\right) N_{d} v(x, y, z) \mathrm{d} x \mathrm{~d} y \mathrm{~d} z .
$$

We first consider the case of $T=0 \mathrm{~K}$ where an analytical solution is existing. We get simple expressions for $\tau, d$, and $v$

$$
\left\{\begin{aligned}
\frac{1}{\tau} & =\frac{\pi}{2} N_{d} \nu_{0} a_{0}^{3} \\
d & =\frac{3}{4} a_{0} \\
v & =\frac{3 \pi}{8} N_{d} \nu_{0} a_{0}^{4} .
\end{aligned}\right.
$$

In Figure 4(a), we compare the results of MC and effective medium simulation in the case of $T=0 \mathrm{~K}$. We observe a good agreement between both simulations for $a_{0}>10 \mathrm{~nm}$ $\left(N_{d}=10^{18} \mathrm{~cm}^{-3}\right)$ which confirms the validity of the effective medium approximation for $a_{0}>\sqrt[3]{N_{d}}$. In contrast, for $a_{0}<\sqrt[3]{N_{d}}$, the effective medium overestimates the speed compared to the MC case.

For a finite temperature, there is no analytical solution for Eqs. (4), (6), and (7). However, it is possible to obtain solutions thanks to numerical integrations. Figure 4(b) shows the numerical estimation in the effective medium approximation of the average speed of exciton with respect to the temperature for different Bohr radii and donor concentrations. For the energy gradient reported in our previous paper $E_{\sigma}=35 \mathrm{meV} / \mu \mathrm{m}$, ${ }^{9}$ we observe a strong decrease of exciton speed above $T=1 \mathrm{~K}$. Depending on the donor concentration, the speed decreases by a factor 3 to 4 from $1 \mathrm{~K}$ to $10 \mathrm{~K}$. The value extracted from time resolved cathodoluminescence experiments ${ }^{9}$ is around $5 \mathrm{~nm} / \mathrm{ps}$ at $10 \mathrm{~K}$. Assuming a donor concentration equal to $N_{d}=5 \times 10^{18} \mathrm{~cm}^{-3}$, it means that the Bohr radius of the donor bound exciton in $\mathrm{ZnO}$ should be close to $a_{0}=5 \mathrm{~nm}$. This value is in good agreement with literature which estimates a Bohr radius of free exciton around $a_{0}=2.34 \mathrm{~nm}$ (Ref. 17) and 1.44 and 3.47 times this value for the Bohr radius of donor bound exciton. ${ }^{18}$ 

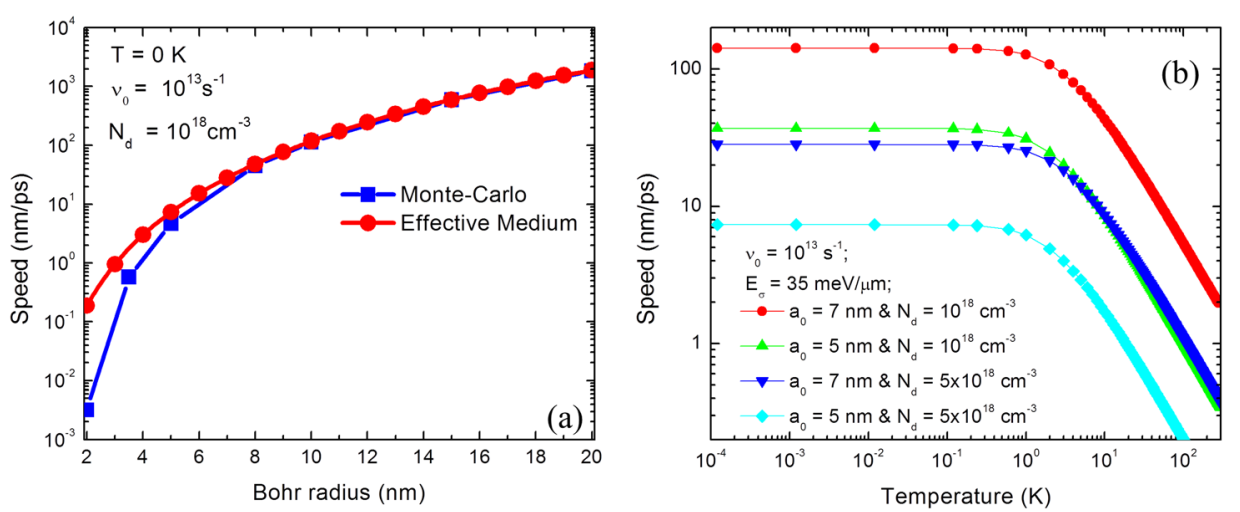

FIG. 4. (a) Average speed of excitons at $T=0 \mathrm{~K}$ as a function of the Bohr radius from MC simulations (blue squares) and analytical model (red dots). (b) Average speed of exciton as a function of temperature for different Bohr radii and donor concentrations with the effective medium model.
However, if we observe a strong decrease of the hopping speed with the temperature, it does not mean that an exciton will not drift at higher temperatures. Indeed, if the hopping process described before governs the dynamics of excitons at low temperature in the case of highly doped semiconductors, others mechanisms are also thermally activated. For instance, when the temperature is increasing, the exciton dynamics will be dominated by the drift of free excitons, where standard exciton mobility mechanisms take place. The transition between the two drift processes (localized to delocalized states) is referred in the literature as the mobility edge. ${ }^{19,20}$

In conclusion, we compute by means of MC simulations the speed of bound excitons in an energy gradient. We show that the mean speed of excitons is strongly dependent to temperature and Bohr radius. Thanks to an effective medium approximation, we deduce an analytical model to estimate the speed of bound exciton at $T=0 \mathrm{~K}$ and we show that this model is valid for $a_{0} \gg 1 \sqrt[3]{N_{d}}$. Finally, we compare our theoretical results with experimental ones and find a good agreement. Our study therefore highlights the relevance of hopping processes in exciton motion at low temperature.

We gratefully acknowledge Xuewen Fu, Dapeng Yu, and Henri Mariette for fruitful discussions. In addition, we acknowledge the financial support from the Swiss National Science Foundation through Project Nos. 129713 and 200020-135003 and from the NCCR Quantum Photonics (Grant No. 128792).
${ }^{1}$ M. L. Lee, E. A. Fitzgerald, M. T. Bulsara, M. T. Currie, and A. Lochtefeld, J. Appl. Phys. 97, 011101 (2005).

${ }^{2}$ R. Banal, M. Funato, and Y. Kawakami, Phys. Rev. B 79, 121308(R) (2009).

${ }^{3}$ G. Jacopin, L. Rigutti, S. Bellei, P. Lavenus, F. H. Julien, A. V. Davydov, D. Tsvetkov, K. A. Bertness, N. A. Sanford, J. B. Schlager, and M. Tchernycheva, Nanotechnology 23, 325701 (2012).

${ }^{4}$ A. Castellanos-Gomez, R. Roldan, E. Cappelluti, M. Buscema, F. Guinea, H. S. J. van der Zant, and G. A. Steele, Nano Lett. 13, 5361-5366 (2013).

${ }^{5}$ J. Feng, X. Qian, C. Huang, and J. Li, Nat. Photonics 6, 866 (2012).

${ }^{6}$ X. Han, L. Kou, Z. Zhang, Z. Zhang, X. Zhu, J. Xu, Z. Liao, W. Guo, and D. Yu, Adv. Mater. 24, 4707 (2012).

${ }^{7}$ B. Wei, K. Zheng, Y. Ji, Y. Zhang, Z. Zhang, and X. Han, Nano Lett. 12, 4595 (2012)

${ }^{8}$ C. P. Dietrich, M. Lange, F. J. Kluüpfel, H. von Wenckstern, R. SchmidtGrund, and M. Grundmann, Appl. Phys. Lett. 98, 031105 (2011).

${ }^{9}$ X. Fu, G. Jacopin, M. Shahmohammadi, R. Liu, M. Benameur, J.-D. Ganière, J. Feng, W. Guo, Z.-M. Liao, B. Deveaud, and D. Yu, "Exciton Drift under Uniform Strain Gradient in Bent ZnO Microwires," ACS Nano (submitted).

${ }^{10}$ H. Mariette, Physica B \& C 146, 286 (1987).

${ }^{11}$ H. Mariette, J. Kash, D. Wolford, and A. Marbeuf, Phys. Rev. B 31, 5217 (1985).

${ }^{12}$ G. Franssen, A. Kamińska, T. Suski, A. Suchocki, K. Kazlauskas, G. Tamulaitis, A. Žukauskas, R. Czernecki, H. Teisseyre, P. Perlin, M. Leszczyński, M. Boćkowski, I. Grzegory, and N. Grandjean, Phys. Status Solidi 241, 3285 (2004).

${ }_{13}^{13}$ A. Müller and M. Grundmann, Phys. Rev. B 87, 035134 (2013).

${ }^{14}$ P. Leroux-Hugon and H. Mariette, Phys. Rev. B 30, 1622 (1984).

${ }^{15}$ V. Ambegaokar, B. Halperin, and J. Langer, Phys. Rev. B 4, 2612 (1971).

${ }^{16}$ A. Miller and E. Abrahams, Phys. Rev. 120, 745 (1960).

${ }^{17}$ R. Senger and K. Bajaj, Phys. Rev. B 68, 045313 (2003).

${ }^{18}$ O. Akimoto and E. Hanamura, J. Phys. Soc. Jpn. 33, 1537 (1972).

${ }^{19}$ M. Oueslati, M. Zouaghi, M. Pistol, L. Samuelson, H. Grimmeiss, and M. Balkanski, Phys. Rev. B 32, 8220 (1985).

${ }^{20}$ N. Mott, J. Phys. C: Solid State Phys. 20, 3075-3102 (1987). 